\title{
qtl.outbred: Interfacing outbred line cross data with the R/qtl mapping software
}

Ronald M Nelson ${ }^{1 *}$, Xia Shen ${ }^{2}$ and Örjan Carlborg ${ }^{1,2}$

\begin{abstract}
Background: qtl.outbred is an extendible interface in the statistical environment, $\mathrm{R}$, for combining quantitative trait loci (QTL) mapping tools. It is built as an umbrella package that enables outbred genotype probabilities to be calculated and/or imported into the software package R/qtl.

Findings: Using qtl.outbred, the genotype probabilities from outbred line cross data can be calculated by interfacing with a new and efficient algorithm developed for analyzing arbitrarily large datasets (included in the package) or imported from other sources such as the web-based tool, GridQTL.

Conclusion: qtl.outbred will improve the speed for calculating probabilities and the ability to analyse large future datasets. This package enables the user to analyse outbred line cross data accurately, but with similar effort than inbred line cross data.
\end{abstract}

\section{Background}

QTL mapping is one of the first steps towards understanding the genetic basis of complex traits. With the rapid advances in genotyping technologies it is common to obtain dense genotype data for many individuals. While there are a number of software packages available for QTL mapping and analysis, none can currently handle large datasets for outbred line crosses. Here, we introduce a software package, qtl.outbred, which provides an interface between outbred line cross data and the popular QTL mapping and analysis tool, R/qtl [1].

qtl.outbred enables the user to convert and import genotype probabilities from outbred line crosses to R/qtl in the freely available $\mathrm{R}$ environment [2]. After importing the genotype probabilities, the functions in R/qtl downstream of its native calc.genprob function can be used on the imported data. qtl.outbred also provides a function for calculating genotype probabilities from outbred line cross data for large datasets using a newly developed and computationally highly efficient algorithm [3]. Alternatively, output from other software, e.g. GridQTL [4], can be ported directly for analysis in R/qtl.

\footnotetext{
* Correspondence: Ronnie.Nelson@slu.se

'Department of Breeding and Genetics, Swedish University of Agricultural

Sciences, Box 7023, SE-75007 Uppsala, Sweden

Full list of author information is available at the end of the article
}

The purpose of this interface is to make the established mapping tools in R/qtl, originally developed for inbred line cross data, available to the wider scientific community. The package focuses on outbred line cross datasets, which are often found in research on agricultural plants and animals, selection lines in experimental species including mice, as well as for a number of animal models of human diseases.

\section{Implementation}

The functions in qtl.outbred are summarized in Table 1. The main task of qtl.outbred is to import genotype probabilities, calculated for outbred cross data, into the R-environment and converting them into an object of the class cross in R/qtl. This data object can then directly be used in the R/qtl package [1] for further QTL mapping analyses. R/qtl is well established and provides a comprehensive set of tools for QTL analysis which are applicable to all type of line cross data (once the genotype probabilities are calculated and imported), but today this software is limited to inbred line cross data. Importing outbred line cross data through qtl. outbred provides access to these tools to users with outbred line cross data.

Calculation of genotype probabilities from outbred line cross data is not trivial and qtl.outbred provides support for obtaining these values. We recommend 
Table 1 Functions in the package qtl.outbred.

\begin{tabular}{ll}
\hline Function & Description \\
\hline calc.prob & Calculating genotype probabilities using the triM algorithm \\
impo.prob & Importing calculated genotype probabilities to R/qtl \\
\hline
\end{tabular}

using the build-in function in qtl.outbred, which is much faster and more accurate than the current methods [5]. This method uses a new algorithm (triM) that calculates genotype probabilities from marker and pedigree data from F2 and back-cross populations, using a hidden Markov model [3].

Other features in qtl.outbred include the option to directly import genotype probabilities generated from the widely used GridQTL software [4]. However, the simple input format used in qtl.outbred, should allow the user to create input files from any other files with genotype probability data.

\section{Results}

qtl.outbred has been extensively tested. Firstly, we established that the triM algorithm produce exactly the same genotype probabilities as R/qtl when inbred line cross data are used (i.e. line crosses of inbred mouse strains). Secondly, we used genotypic data from an outbred line cross between domesticated and wild chickens with a simulated phenotype. Genotype probabilities were calculated with the triM algorithm using qtl.outbred to interface it with $\mathrm{R} / \mathrm{qt}$. The single- and two-QTL genome scan for this dataset is illustrated in Figure 1. The identified peaks correspond to where the QTL were simulated. Lastly, we calculated QTL

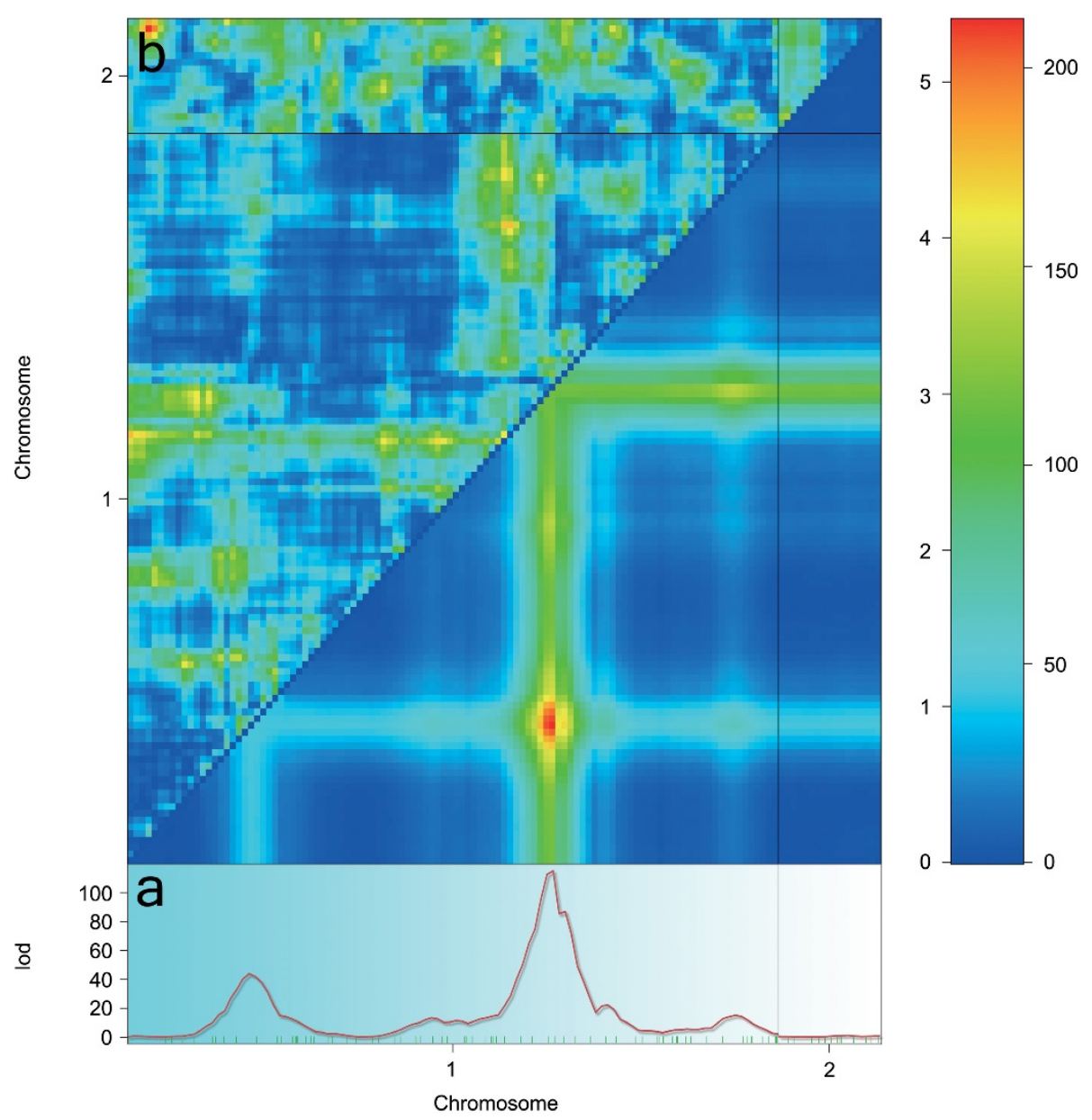

Figure 1 The graph was obtained by using outbred line cross data (domesticated and wild chicken intercross genotypic data with simulated phenotype), calculating genotype probabilities with the triM algorithm from the qtl.outbred interface and importing it directly to R/qtl where the genome scans were performed. LOD scores for Haley-Knott regression [6] for (a) single-QTL genome scan and (b) two-QTL genome scan are reported. LOD scores are indicated on the colour scale where, numbers to the left correspond to the upper triangle indicating two-locus epistasis and values to the right correspond to the lower triangle indicating the significance for a test of two versus one QTL. 
genotype probabilities for the simulated chicken intercross using GridQTL. These genotype probabilities were imported in R/qtl, using the qtl.outbred interface, and the conducted QTL scan gave similar results to those reported in Figure 1.

\section{Conclusion}

The purpose of qtl.outbred is to: 1. serve as an interface between the established software packages GridQTL and $\mathrm{R} / \mathbf{q t l} ; 2$. provide an alternative to calculate QTL genotype probabilities in outbred crosses faster and more accurate than current software via the triM algorithm, and interface the results with R/qtl; 3. enable genotype probabilities calculated via any other method for outbred line cross to be imported into R/qtl; 4. provide these functions in a user friendly environment. This package is designed to fill the need for a fast and efficient QTL mapping environment for large datasets for outbred line crosses.

\section{Availability and Requirements}

Project name: qtl.outbred

Project home page: https://r-forge.r-project.org/R/? group_id $=844$

Operating systems: Windows, Unix-like (Linux, Mac OSX)

Programming language: $\mathrm{R}, \mathrm{C} / \mathrm{C}++$, Perl

Other requirements: $\mathrm{R}$, Perl

License: GNU GPL

Any restrictions to use by non-academics: None.

\section{Acknowledgements}

This work was supported by the Future Research Leader program of the Swedish Foundation for Strategic Research and a EURYI Award from the European Science Foundation to ÖC. We thank Carl Nettelblad and Lucy Crooks for access and assistance with the triM algorithm.

\section{Author details}

'Department of Breeding and Genetics, Swedish University of Agricultural Sciences, Box 7023, SE-75007 Uppsala, Sweden. ${ }^{2}$ Department of Cell and Molecular Biology, Uppsala University, BMC Box 598, SE-75124 Uppsala, Sweden.

\section{Authors' contributions}

RMN, XS and C developed the concept. RMN and XS wrote the software and performed data analysis. RMN and ÖC wrote the manuscript. All authors read and approved the final manuscript.

\section{Competing interests}

The authors declare that they have no competing interests.

Received: 10 February 2011 Accepted: 26 May 2011

Published: 26 May 2011

\section{References}

Broman KW (2003) R/qtl: QTL mapping in experimental crosses. Bioinformatics 19:889-890. doi:10.1093/bioinformatics/btg112.

R Development Core Team (2010) R: A Language and Environment for Statistical Computing.
Nettelblad C, Holmgren S, Crooks L (2009) cnF2freq: Efficient Determination of Genotype and Haplotype Probabilities in Outbred Populations Using Markov Models. Proceedings of the International Conference on Bioinformatics and Computational Biology. Las Vegas, Nevada, USA; 13-16 July 2009. SpringerVerlag pp 307-319

Seaton G, Hernandez J, Grunchec JA, White I, Allen J, de Koning DJ, Wei W, Berry D, Halley C, Knott SA (2006) GridQTL: a grid portal for QTL mapping of compute intensive datasets. Proceedings of 8th World Congress on Genetics Applied to Livestock Production. 13-18 August 2006. Belo Horizonte, MG, Brasil

Crooks L, Nettelblad C, Carlborg Ö An improved method for estimating chromosomal line origin in QTL analysis of crosses between outbred lines. G3: Genes, Genomes, Genetics (in press)

Knott SA, Haley CS (1992) A simple regression method for mapping quantitative trait loci in line crosses using flanking markers. Heredity 69:315-324

\section{doi:10.1186/1756-0500-4-154}

Cite this article as: Nelson et al:: qtl.outbred: Interfacing outbred line cross data with the R/qtl mapping software. BMC Research Notes 2011 4:154.

\section{Submit your next manuscript to BioMed Central and take full advantage of:}

- Convenient online submission

- Thorough peer review

- No space constraints or color figure charges

- Immediate publication on acceptance

- Inclusion in PubMed, CAS, Scopus and Google Scholar

- Research which is freely available for redistribution 